\title{
CORRIGENDUM \\ Efficient delivery of lentiviral vectors into resting human CD4 T cells
}

X Geng, G Doitsh, Z Yang, NLK Galloway and WC Greene

Gene Therapy (2016) 23, 320-322; doi:10.1038/gt.2015.115

Correction to: Gene Therapy (2014) 21, 444-449; doi:10.1038/gt.2014.5

Figure 1c and Figure $2 \mathrm{~b}$ of our previously published paper are incorrect as presented. The experiments were independently repeated with multiple donors and the new data are now presented as Figure 1c, Figure 1d and Figure 2b. The original conclusions drawn from these figures and the techniques described in the full manuscript remain the same. The VLP-Vpx technology provides an efficient and reliable method for introducing lentiviral shRNAs into resting CD4 T cells, especially into primary blood-derived lymphocytes that are in a deep resting state.

The authors apologize for not detecting the error in these two figures prior to publication. 
a Vpx-incorporated
Virus-like Particles (Vpx-VLPs) irus-like Particles (Vpx-VLPs)

Fresh Isolated CD4 T Cells

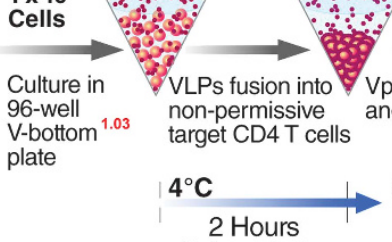

Spinoculation

b

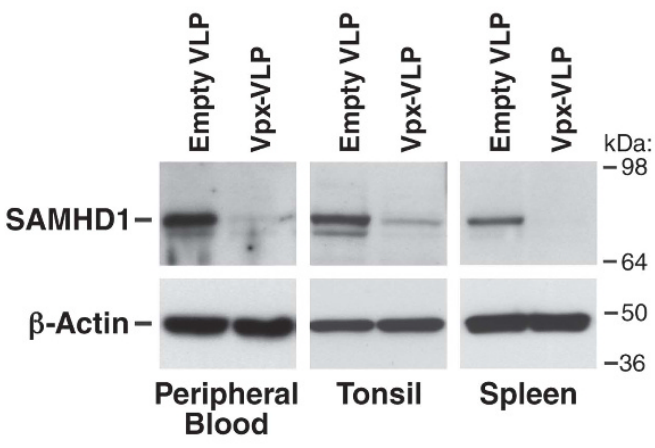

mCherry-Reporter shRNA-coding HIV-1 Lentivirus 

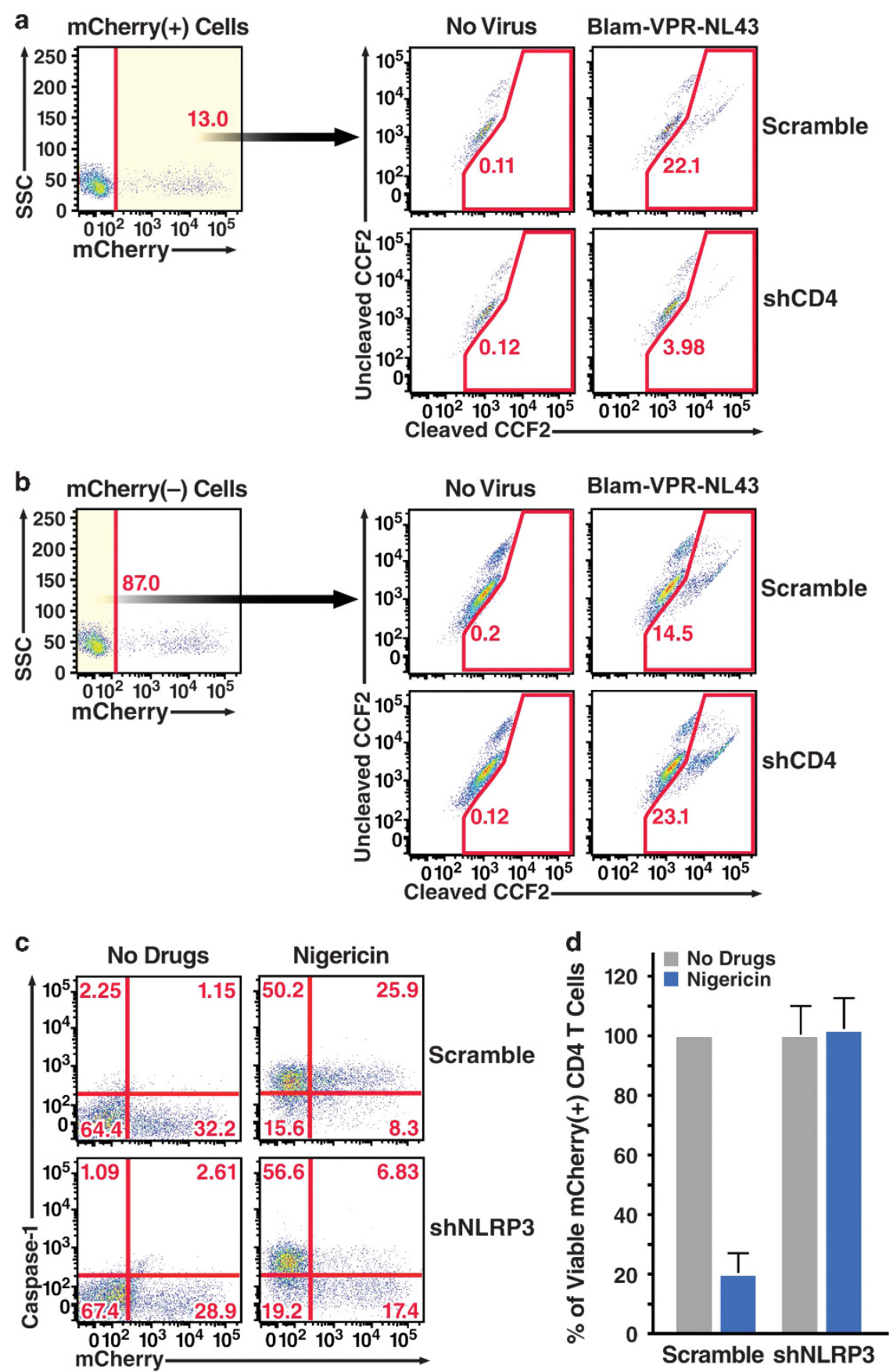

Figure 2. Modulating specific CD4 T cells responses by HIV LV (a, b) Infection of shCD4 HIV LV impedes HIV-1 fusion to resting CD4 T cells isolated from blood. To test the ability of HIV-1 to fuse with infected cells, we used an HIV virion-based fusion assay that measures BlaM activity delivered to target cells upon the fusion of X4-tropic NL4-3 strain of HIV-1 containing BlaM fused to the Vpr protein (BlaM-VprNL4-3). ${ }^{45}$ Inhibition of fusion was specifically observed in the mCherry-positive (a), but not in the mCherry-negative (b) cells residing in the same cultures. (c) Infection of shNLRP3 HIV LV blocks caspase-1 activation in tonsillar CD4 T cells treated with nigericin. (d) Infections with shNLRP3 HIV LV prevent caspase-1-mediated death of mCherry-positive CD4 T cells treated with nigericin. Inhibition of cell death was not inhibited by mCherry-positive Scramble HIV LV. 